\section{Trends in rate of acute myocardial infarction among patients aged $<30$ years}

\author{
Xiaofan Guo, Zhao Li, Eric Vittinghoff, Yingxian Sun and Mark J. Pletcher
}

We read with great interest the excellent Review by Andersson and Vasan (Epidemiology of cardiovascular disease in young individuals. Nat. Rev. Cardiol. http://dx.doi.org/ 10.1038/nrcardio.2017.154; 2017) ${ }^{1}$. The authors discussed the high burden of cardiovascular disease risk factors and the worrisome trends of ischaemic heart disease, heart failure, atrial fibrillation, and sudden death in young individuals, highlighting a new epidemic of cardiovascular disease in this segment of the population. However, the Review focused mainly on the population aged $>35$ years, especially for acute myocardial infarction (AMI), owing to scarce data. In clinical practice, we have diagnosed patients aged $<30$ years with AMI more frequently than ever before, although AMI still remains rare in these young adults. Therefore, we want to discuss our recent findings on this topic further.

We obtained national data from the Healthcare Cost and Utilization Project Nationwide Inpatient Sample administrative

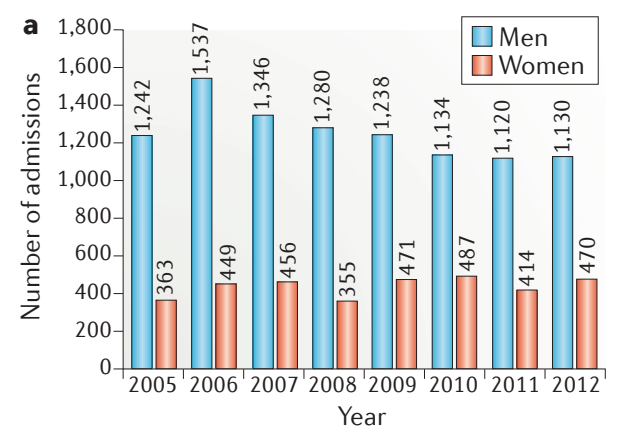

Figure 1 | Trends in adult patients aged $<30$ years with acute myocardial infarction in the USA. a | Annual number of admissions. b | Annual mortality. Sampling weights were used to produce nationally representative estimates.

file in the USA ${ }^{2}$. Using these data, we identified 2,832 hospitalizations with a principal discharge diagnosis of AMI among adult patients aged $<30$ years from 2005 to 2012 , representing an estimated 13,492 AMI hospitalizations nationally after applying sampling weights. The prevalence of smoking, obesity, and heart failure among these young patients increased significantly from 2005-2008 to 2009-2012. As shown in FIG. 1, the annual number of AMI hospitalizations in young women increased from 363 to 470 cases during 2005-2012, with no decline in in-hospital mortality. Annual admissions among men reduced from 1,242 to 1,130 cases during 2005-2012, but in-hospital mortality increased significantly from $0.9 \%$ to $2.7 \%$ during the same period ( $P=0.025$ for trend). An increasing trend of percutaneous coronary intervention utilization was observed for both sexes $(P<0.001$ for trend in both sexes). An increased trend in in-hospital mortality was also observed in all patients from 2010 onwards (FIG. 1).

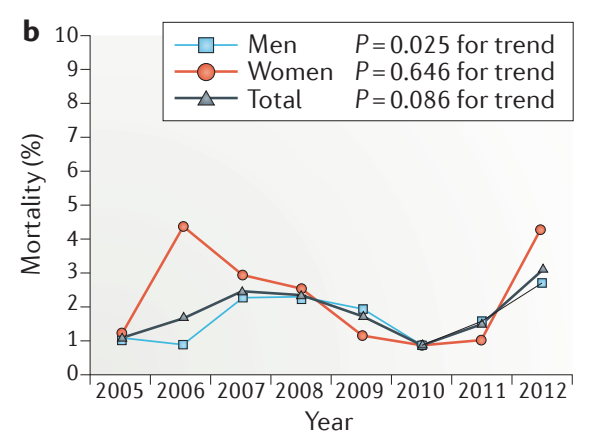
\section{a.}

\author{
The
}

Epidemiological data on AMI among adults aged $<30$ years have been reported in only a few case studies ${ }^{3,4}$. However, the disease is not as rare in this population as previously thought. As mentioned in the Review by Andersson and Vasan ${ }^{1}$, previous studies have shown a gradual reduction in AMI events in patients aged 35-64 years ${ }^{5}$, whereas Gupta and colleagues ${ }^{6}$ reported an absence of significant reductions in hospitalization rates among patients aged $<55$ years, indicating a potential shift of AMI to the younger population. Our findings suggest that this shift has even spread to very young adults aged $<30$ years, who show suboptimal outcome despite increased utilization of percutaneous coronary intervention. We believe more attention should be focused on young adult patients aged $<30$ years at risk of AMI.

Xiaofan Guo, Zhao Li, and Yingxian Sun are at the Department of Cardiology, the First Hospital of China Medical University, 155 Nanjing North Street, Heping District, Shenyang, Liaoning, China.

Xiaofan Guo, Eric Vittinghoff, and Mark J. Pletcher are at the Department of Epidemiology and Biostatistics, University of California-San Francisco,

550 16th Street, San Francisco, California, USA.

Zhao Li is at the School of Medicine, Johns Hopkins University, 720 Rutland Avenue, Baltimore, Maryland, USA.

Correspondence to X.G. guoxiaofan1986@hotmail.com doi: 10.1038/nrcardio.2017.191 Published online 14 Dec 2017

1. Andersson, C.\& Vasan, R. S. Epidemiology of cardiovascular disease in young individuals. Nat. Rev. Cardiol. http://dx.doi.org/10.1038/nrcardio.2017.154 (2017).

2. Healthcare Cost and Utilization Project. Facts and figures: statistics on hospital-based care in the United States, 2009. HCUP-US https://www.hcup-us.ahrq.gov/ reports/factsandfigures/2009/TOC 2009.jsp (2009).

3. Weinberger, I. et al. Myocardial infarction in young adults under 30 years: risk factors and clinical course. Clin. Cardiol. 10, 9-15 (1987).

4. Falcone, M. W., Grayburn, P. A. \& Roberts, W. C. Acute myocardial infarction at 25 years of age. Proc. (Bayl. Univ. Med. Cent.) 17, 363-365 (2004).

5. Degano, I. R. et al. Twenty-five-year trends in myocardial infarction attack and mortality rates, and case-fatality, in six European populations. Heart. 101, 1413-1421 (2015)

. Gupta, A. et al. Trends in acute myocardial infarction in young patients and differences by sex and race, 2001 to 2010. J. Am. Coll. Cardiol. 64, 337-345 (2014).

mpeting interests statement 